\title{
Ultrasound-guided Erector Spinae Plane (US- ESP) Block associated to Dexmetomidine cooperative sedation for anesthetic management in breast cancer surgery: a case report .
}

$\underline{\text { C. Giordano }}^{1}$, S. Ranocchia 2 , D. Nicolotti 1 , P. Fusco ${ }^{3}$, V. Danzi ${ }^{1}$, P. Scimia 1.

${ }^{1}$ Hospital of Cremona, Anesthesia and Intensive Care Unit, Cremona, Italy.

2University Hospital of Parma, Anesthesia and Intensive Care Unit, Parma, Italy.

${ }_{3}^{3}$ S.Salvatore Academic Hospital of Aquila, Anesthesia and Intensive Care Unit, Aquila, Italy.

\section{Background and Aims:}

Ultrasound-guided Erector Spinae Plane Block (US-ESPB) is a recently described regional block technique for anesthesia and analgesia of the chest wall. Dexmetomidine (DEX) is an $\alpha-2$ agonist that can provide cooperative sedation during surgery. We reported a case of US-ESPB associated to intraoperative DEX sedation in a patient undergoing breast cancer surgery.

Methods:

A 42 years-old patient, ASA 2, was scheduled to undergo quadrantectomy with sentinel lymph node biopsy. Before surgery, we performed a US-ESPB and $20 \mathrm{ml}$ of 0,5\% Ropivacaine were injected using a $90 \mathrm{~mm}$ needle (Temena $\left.{ }^{\circledR}\right)$ deep to the erector spinae muscle and superficial to T5 transverse processes. The patient received intravenously DEX in a loading dose of $1 \mathrm{mcg} / \mathrm{kg}$ over 15 minutes, followed by an infusion of $0,4 \mathrm{mcg} / \mathrm{kg} / \mathrm{h}$ and oxygen (4 l/min) was administered by facemask.

\section{Results:}

We obtained adequate surgical anesthesia and a good quality postoperative analgesia. Moreoverer, DEX infusion provided cooperative sedation during surgery (Ramsay 3) without causing respiratory depression of the patient. Only $3 \mathrm{~g}$ of acetaminophen were administered postoperatively. No complications were recorded.

\section{Conclusions:}

This case report suggested that US-ESPB associated to intraoperative DEX cooperative sedation could represent a reliable strategy for anesthetic management in breast surgery.

Keywords:

Ultrasound-guided Erector Spinae Plane (US- ESP) Block, Dexmetomidine sedation,breast cancer surgery. 